Marek Kośny*

DOI: $10.2478 /$ v10241-012-0004-8

\title{
Reference groups and complaints about inequality
}

\section{ABSTRACT}

Although influence of reference population on assessment of individual position is well recognized on the ground of sociology, its impact is rarely taken into account in economic analysis of inequality. Few analyses that are concerned with issue of reference groups in the context of inequality and relative deprivation measurement deal, however, with single reference group. The aim of the paper is consideration on how concept of reference populations of different kind (reference groups and reference categories) may be applied to assessment of aggregated complaint that results from inequality in income (wealth) distribution.

KEYWORDS: relative deprivation, inequality, reference groups

\section{INTRODUCTION}

Over past few decades, processes of globalization drew economists' and sociologists' attention to the problems of inequality, poverty and - treating the issue more generally - asymmetry in the distribution of endowments and outcomes. Significance of

* Correspondence regarding the paper should be sent to: Dr Marek Kośny, Wroclaw University of Economics, ul. Komandorska 118/120, 53-345 Wroclaw, email: marek.kosny@ue.wroc.pl 
research in this area is undoubtedly confirmed by a Nobel Prize in economic sciences, awarded in 1998 to Professor Amartya Sen 'for his contributions to welfare economics'.

On account of its complexity and interdisciplinary character, the notion of inequality is analyzed from the point of view of many fields of science, particularly economy and sociology. However, "with respect to issues of measurement and operationalization, there appears to be rather little in the way of disciplinary cross-fertilization, despite the evidence of convergence in the conceptual challenges and problems that have been identified in each discipline" Grusky and Kanbur (2005).

In fact, on the ground of sociology, it is quite clear that one of the most important issues taken into account by an individual, while assessing the position in the society and satisfaction from this position, is the reference group. Mainstream inequality analysis in the field of economics concentrated on the approach - related to the utilitarian sight of welfare - which negated interdependences between utility functions of individuals (see, for instance, the seminal paper of Atkinson (1970); although some authors - for instance Sen (1973) - argued that social welfare function of a standard, additive type is not the only one that can underlie inequality measurement).

The idea of reference groups was reflected in researches carried out on relative deprivation. This notion, being closely related to inequality, is much more concerned with the situation of individuals, as 'inequality is the cause of the feeling of relative deprivation' Podder (1996, p. 359). Instead of objective disparities in distribution of wealth, subjective perception is being analyzed. As pioneering in this field could be recognized work of Runciman (1966), but the issue seems to have attracted much more attention over last years - see Grusky and Kanbur (2004), Podder (1996) and Pedersen (2004). A ground of this current interest could be the fact that in many applications, where 'stand- 
ard' inequality measures are being used, the application of their more feelings-oriented counterparts would be more much appropriate. One can present the theory of taxation as an example of such field. The framework based on the sociological idea of reference groups, adds some arguments to the discussion on concept of progression in income taxation by defining an optimal - with respect to relative deprivation (basing on subjective evaluation of income differences) - income distribution. In this way, changing the general approach may influence the choice of desired target of tax system potential reforms by enabling to discover another - different from ideal equality - direction for improving perception of justice of the system. Some considerations on it will be presented in the fourth section.

The presented approach to the measurement of relative deprivation will extensively draw from the concept of complaint - see Temkin (1986 and 1993). Original proposal of Temkin concerns measurement of inequality - objective character of complaints, not connected with the feeling of injustice with respect to inequality, is clearly emphasized both by the author and by others, developing his concept - see Cowell and Ebert (2004, p. 7374). Notion of complaint seems to be easily interpretable in the feelings-oriented framework, which results in the measure of relative deprivation.

In this context, the primary aim of this paper is presenting some hypotheses concerning the shape of the complaint function of an individual. Then this function will be used as a basis for construction of appropriate measure of relative deprivation.

The structure of the paper is as follows. The second section will present some general hypotheses - proposed within the framework of reference groups - on properties of the proposed measure of relative deprivation. In the third section, after formulating detailed assumptions, an example of such measure will be constructed. In the fourth section, there will be shown some consequences of changing the point of view on issue of inequality 
- especially in the context of evaluation of the tax systems and quest for optimal (with respect to aggregate complaint) level of inequality. The last, fifth section concludes.

\section{REFERENCE GROUPS AND PERCEPTION OF INEQUALITY}

As stated above, the concept developed in the next of this paper will draw from the Temkin's (1986 and 1993) idea of inequality, measured by counting complaints. Temkin defines his general attitude to inequality by stating that "to say that the best-off have nothing to complain about is in no way to impugn their moral sensibilities. They may be just as concerned about the inequality in their world as anyone else. Nor is it to deny that, insofar as one is concerned about inequality, one might have a complaint about them being as well off as they are. It is only to recognize that, since they are at least as well off as every other member of their world, they have nothing to complain about. Similarly, to say that the worst-off have a complaint is not to claim that they will in fact complain (they may not). It is only to recognize that it is a bad thing (unjust or unfair) for them to be worse off than the other members of their world through no fault of their own" (Temkin, 1986, p. 102).

He proposes an approach to measurement of inequality, which is, in fact, strictly related to measurement of relative deprivation (but, what is underlined by Cowell and Ebert (2004, p. 74), not equivalent). This close relationship to the relative deprivation has its source in interpretation of inequality as aggregation of individual complaints - Temkin defines the aim of his paper as consideration on 'how bad the inequality in a situation is from the standpoint of particular individuals in that situation' Temkin (1986, p. 102). For this reason, complaints are understood as dissatisfaction with partition of goods (income) within the population and, therefore, only complaints with respect to inequality 
- what is explicitly stated by Temkin - are taken into account (also in this paper complaints are said to be generated solely by inequality).

In presented approach, complaints will be measured with respect to income - such simplification enables to omit the problem of welfare measurement and does not affect provided arguments (further discussion on choice of income inequalities as source of complaints will be presented in the next of this paper). This choice may be found to be in accordance with original concept of Temkin. He is concerned with inequalities of welfare, but declares that his approach may be expressed in terms of other endowments or outcomes - see Temkin (1986, p. 100).

In his works Temkin $(1986,1993)$ discusses two aspects of inequality measurement. Firstly, he is concerned with specification of the reference income that is chosen by an individual and defines the extent of complaint. He distinguishes three possibilities:

- income of the person that is the best-off (BOP),

- incomes of all persons that are better-off (ATBO),

- average income in the population (AVE).

Secondly, he indicates three ways of aggregation of individual complaints over population:

- calculating the mean complaint,

- calculating the weighted mean of complaints (where higher weights are attributed to higher values of complaint - certain weighting scheme is not discussed by Temkin),

- taking into account only the value of the maximal complaint in the population - application of Rawls' concept of justice seer Rawls (1971).

However, he leaves open the issue of individual complaint's extent. He just assumes that individual complaint results from difference in welfare levels (that of individual and the referential one). This simple idea is extended by Cowell and Ebert (2004) in order to give the resulting class of inequality measures, the 
desired axiomatic properties. Nevertheless, even in this case, function of individual complaint is increasing with the welfare (income) difference. Such relationship could be seen as conventional in the literature on inequality measurement (see Atkinson (1970), Cowell (2000), Lambert, (2001), Yitzhaki (1998) on design of most popular inequality measures).

This standard assumption does not take into account the sociological evidence about existence of reference groups and their impact on the subjective evaluation of welfare (income) level even though the concept of reference groups itself is considered by Cowell and Ebert (2004).

Despite the fact that idea of considering of this notion in inequality measurement is not new (the approach first appeared in Runciman (1966) in the context of relative deprivation), concrete applications were proposed by assumption that inequality (relative deprivation) is measured within the reference group - see Pedersen (2004), Podder (1996). Certainly, these reference groups had been defined in broad outline and encompassed, for instance, the whole society (country, nation). It is not to deny that the whole population of the given country, constitutes some form of the reference group for all the members of that state because of the common heritage, currency, law, public statistics and so on. However, such a group could not be taken as a group in the sociological sense. Merton (1957 (2002)1 , p. 350-351), in his definition of reference group, as a characteristic (distinguishing it from reference category) gives the necessity of personal interactions between the group members. Due to his classification, groups like, for example, nations or social classes could be classified as reference categories. This differentiation does not exhaust essence of both notions: to be able to define given subpopulation as a reference group or reference category for a given individu-

${ }^{1}$ All quotations from this book (page numbers) will be given according to Polish translation, published in 2002 by PWN, Warszawa. 
al, there need to be fulfilled certain additional conditions. These conditions directly depend on kind of reference population that is being dealt with. From the point of view of this paper - in the context of perception of inequality - of a special interest will be comparative reference groups. Such groups embrace all the people that constitute point of reference, which enables a given person to assess his/her position in the population (wealth, income) in comparison to the position (wealth, income) of others - see Merton (1957 (2002), p. 336).

That breeds the problem of defining the reference group in the way that would be useful in the assessment of the relative deprivation. The earlier mentioned differentiation, suggested by Merton, results in recommendation that reference group should be rather small. However, in the context of statistical analyses, which involves dealing with very large groups with anonymous members, it is sometimes unrealistic assumption and could not be considered as indisputable characteristic. Such analyses, dealing with very large populations, make it necessary to define reference groups arbitrarily. Despite limited precision of such conduct - only some well structuralized groups can be precisely modeled; modeling of individual reference groups is impossible because social relations' complexity, see Merton (1957 (2002), p. $300)$ - there exists a possibility of specifying some general assumptions.

First of all, it could be stated that income (material status) acts as one of the most important factors, shaping contemporary societies; especially - resulting from the income - possibility to satisfying needs through consumption - see Belk (1985). In this way, many aspects of social activity within society are significantly influenced by income-related characteristics of an individual (further considerations on relationship between income, expenditures and wealth will be presented in the next of this section). Class-based society, which dominated in previous centuries and where membership of a certain class was de- 
termined by birth, transformed in contemporary societies into society based on classes that are distinguished with respect to income. As a consequence of this transformation, there could be observed changes in behavioral patterns: people possessing an approximate income, tend not only to buy similar goods but they also choose similar places of residence or even favorite pastimes (generally speaking, they unify consumption patterns that are at the heart of the materialistic world). This process, in a natural way, results in reference groups, distinguished on basis of income (wealth). In no way does it restrict existence of other reference groups, distinguished with respect to other factors. However, many of such groups - for instance groups of people with similar education level or job (especially seen in the context of age) - often involve also approximate level of income.

Secondly, without loss of generality, it may be assumed that there exists only one reference group, distinguished with respect to a given factor (e.g. income) - analysis would easily be extended to the case of many such groups. Taking into account an arbitrary and rough character of the approach, distinguishing of other potential groups can be carried out through broadening the initial group. This method could be applied especially when differentiating between types of reference groups. As a basis one can take the concept of comparative reference group; on types of reference groups see, for example, Merton (1957 (2002), p. $336 \mathrm{ff}$.) or Dawson and Chatman (2001). Extending the analysis on, for instance, normative reference groups would result in apparent increase in the extent of the reference group.

Thirdly, as indicated above, there will be distinction between two kinds of reference populations for the individual (it can also be interpreted as levels of reference group): reference group and reference category. While members of reference group are treated individually (the direct interaction would be the ideal situation), reference category is said to define a general reference point. This reference point is fixed by the average income 
(wealth), and overall impact of the category may be measured (weighted) by its size. Also in this case, there could be defined more than one reference category - for example, if aggregation level is taken into account, there could be distinguished categories such as local community, nation, race and so on. However, it requires precise definition of each of them and precise assessment of its importance in shaping of complaint and relative deprivation. In a general case that is analyzed in this paper, it will be assumed that every person that does not belong to the reference group, is a member of a single reference category (covering the rest of analyzed population), and that these two sub-populations do not overlap.

The last assumption concerns the choice of the analyzed factor. So far, mainly the notions of income or wealth have been used. Nevertheless, that choice is not evident. Analyzing individual complaints, it may be stated that they are made about expenditure, not income (or changes in them - but this case will not be further analyzed in this paper). The simple assumption, underlying such statement, is that expenditure is much easier observable. Decrease in income of reference person does not reduce feeling of dissatisfaction (complaint) until it is reflected in lowering of standard of living (expenditure). Moreover, what is often emphasized in researches on wealth and its determinants, expenditure is much more stable over time.

It is also inappropriate to use here the argument given by Podder (1996, p. 357) that differences in consumption (expenditure) are not a good measure of dissatisfaction, because of consumption patterns differences between groups. Narrowing the direct comparisons to the reference group significantly reduces such incompatibilities.

However, it is not to deny that in longer period, income (in a broad sense) is generally fundamental both for expenditure and wealth. That is probably why income is most often analyzed in the context of inequality. Because of this common usage and - 
presented in the last part - application to personal income tax, income inequality will also be analyzed in this paper. However, what has been already suggested, income could be easily exchanged with wealth or expenditure - especially in the analyzed framework, based on assessing complaints.

The above mentioned structure of reference populations directly influences assessment of individual complaints. With respect to income differences, there can be distinguished two (in an elementary case that is analyzed in this paper) or more such reference populations.

The first one covers reference group. Because of personal interactions, all differences are overestimated: slightly higher income of a neighbor, a co-worker or an acquaintance generates complaint comparable (or even higher) than in case of much wealthier, but anonymous person. Therefore, the extent of complaint rapidly increases with income difference and attains maximum at the border of the reference group. In this way income of other individuals, belonging to the same reference group, influences income evaluation (satisfaction) much stronger than those not belonging to this group.

The second reference population could be characterized as reference category. Lack of personal interactions causes that an individual perceives this group from the angle of average income of its members. More, it diminishes feeling of complaint. The extent of individual complaint attains the local minimum for individuals that are just outside the reference group and increases with income difference (but much slower than in reference group). The overall impact of this group on complaint of the individual is additionally influenced by this group's size.

Such differentiation denotes correlation with two concepts of shaping the extent of individual complaint in the Temkin's theory. Within the reference group, due to interactions, complaints will depend on income of all those, who are better-off (ATBO). Income satisfaction depends not only on average income in ref- 
erence group, but on the whole distribution of income within this group - see Merton (1957 (2002), p. 330, footnote 1). Thus an individual is said to take into account income of all people (separately). Only those that are better-off are considered, because - following Temkin - analysis involves no moral sensibilities (that could make rich ones concerned with the problem of inequality - see the beginning of this section). And there is assumed that complaints with respect to inequality arise only in comparisons with wealthier individuals. In particular, the problem of complaints that arise as a result of comparison with less wealthy persons (for example in the situation when they are thought not to deserve even such income) will not be dealt with in this paper - such kind of complaint could not be treated as a result of income inequality itself.

Outside the reference group complaints arise in relation to the observed average income. This generally relates to one of concepts - AVE - considered by Temkin; with two exceptions, however. Firstly, only income of people that are better-off is taken into account while calculating the average income in the reference category - people that are worse-off are just not the members of the reference category (understood in the previously characterized manner). Secondly, the class of location measures should not be restricted only to arithmetic (weighted or not) mean. The individual is said to have only general view of this reference category and, as a consequence, does not know the exact income distribution. And this lack of information (combined with presence of asymmetry, which is generally significant and intrinsically bound up with distribution of income) causes that, in fact, some other location measures may be more appropriate, for instance, mode or median. 


\section{EXAMPLE OF APPLICATION}

The described framework can be used to construct a relative deprivation measure. The one that will be presented in the next of this section should be taken only as an example of how described ideas could be translated into an appropriate index. Lack of exact empirical results from the field of psychometrics makes this example quite arbitrary and it needs further research to make this measure reflecting actual situation.

Construction of the index of relative deprivation involves three-stage procedure.

1. Modeling the function of individual complaint (complaint of an individual that results from comparison with other individual that is better-off or from comparison with average income of individuals from reference category).

2. Defining the method of aggregation of complaints for a given individual (assessing the aggregate complaint of an individual that results from inequality in income distribution).

3. Defining the method of aggregation of complaints over individuals in the population (assessing the aggregate - or mean - complaint with respect to inequality in income distribution).

\section{1) Complaint function}

The most fundamental issue in the presented approach is the construction of the function that determines the extent of individual complaint (of one person with respect to the other that is betteroff). Changes in two other stages, dealing with appropriate aggregation of such complaints (for instance weighting schemes), can often be substituted by suitable modifications of this function.

Some general considerations, presented in previous section, enable to formulate number of assumptions that should be met by the complaint function; for analogous sets of assumptions see 
Podder (1996), Cowell (2000) or Cowell and Ebert (2004). Clearly, the following assumptions are special cases of all possible formulations. Assumptions that are of fundamental importance for the approach are, therefore, signed with asterisk.

Assumption $1^{*}$. Complaints arise as a consequence of inequalities in income distribution.

Assumption 2*. Individual has a complaint only with respect to people that are better-off (with higher income).

Assumption 3. Extent of individual complaint is scale invariant.

Assumption 4. Complaint function (C) fulfills number of technical requirements.

a. Anonymity.

Income distribution in analyzed population is the only characteristics, taken into account in assessment of complaints extent: no other characteristics - besides income - influence value of a complaint function.

b. Continuity.

If it is possible to assess precisely the extent of reference group, this requirement could be overruled - in such a case, at the end of reference group, discontinuity would be supposed to occur.

c. Normalization*.

It is assumed that to a maximal level of dissatisfaction (complaint) is assigned a certain number (in the following of this paper -1) - such a complaint arises when comparing with the best-off person in the reference group. Under assumption that maximum level of dissatisfaction is attained while comparing with the best-off person in the whole population, it could be accepted that this value tends to infinity (such change would significantly influence properties of the resulting relative deprivation measure). Minimum of complaint function (in this paper 0 ) is attained for equal incomes. 
Assumption $5^{*}$. Complaint function reflects differences between reference group and reference category.

a. Within reference group, the function increases with income difference.

b. For incomes just above the reference group function value is decreasing to the local minimum (in case of discontinuity see Assumption $4 \mathrm{~b}$ - this part is not present). This local minimum could be characterized as an area of 'maximum indifference'.

c. Function once again increases with income difference in reference category. It asymptotically attains 1 for infinitely large income differences.

The example of a function that reflects extent of complaint and meets described requirements is given by formula:

$$
C\left(x_{i}, x_{j}, \alpha, \beta\right)=1-\left|\frac{2 \alpha+1}{f\left(x_{i}, x_{j}, \alpha, \beta\right)}-\alpha\right|-\alpha \mid
$$

where $x_{i}, x_{j}$ denotes incomes of persons, respectively, $i$ and $j$ and $x_{j} \geq x_{i}>0$. Parameter $\alpha$ defines extent of complaint that is attained in the local minimum of $C$ and therefore $1-\alpha$ may be interpreted as impact of membership in reference group on extent of complaint $(0 \leq \alpha<1)$. Parameter $\beta>1$ describes size of reference group $\left(\beta x_{i}\right.$ denotes maximal income in person's $i$ group of reference). Function $f$ is a measure of income difference between persons $i$ and $j$ that fulfill conditions $\underset{x_{j} \geq x_{i}}{\forall} f\left(x_{i}, x_{j}, \alpha, \beta\right) \geq 1$, $\partial f\left(x_{i}, x_{j}, \alpha, \beta\right) / \partial\left(x_{j}\right)>0$ and $\partial f\left(x_{i}, x_{j}, \alpha, \beta\right) / \partial\left(x_{i}\right)<0$. In the simplest case function $f$ could be defined as:

$$
f\left(x_{i}, x_{j}, \alpha, \beta\right)=\frac{x_{j}}{x_{i}},
$$


however, in such a case it is not possible to define explicitly extent of the reference group (parameter $\beta$ ). To omit this limitation, definition of $f$ could be extended to the following form:

$$
f\left(x_{i}, x_{j}, \alpha, \beta\right)=\frac{1}{2 \alpha(\beta-1)}\left(\frac{x_{j}}{x_{i}}-1\right)+1
$$

Function $f$ need not to be linear in quotient of $x_{i}$ and $x_{i}$. It may take other forms that fulfill conditions given above (for instance exponential or logarithmic function of this quotient). Making such corrections demands additional justification concerning intensities of changes in feelings of complaint in relation to changes in income differences.

The graph of complaint function with income difference given by equation (3) is plotted on Figure 1.

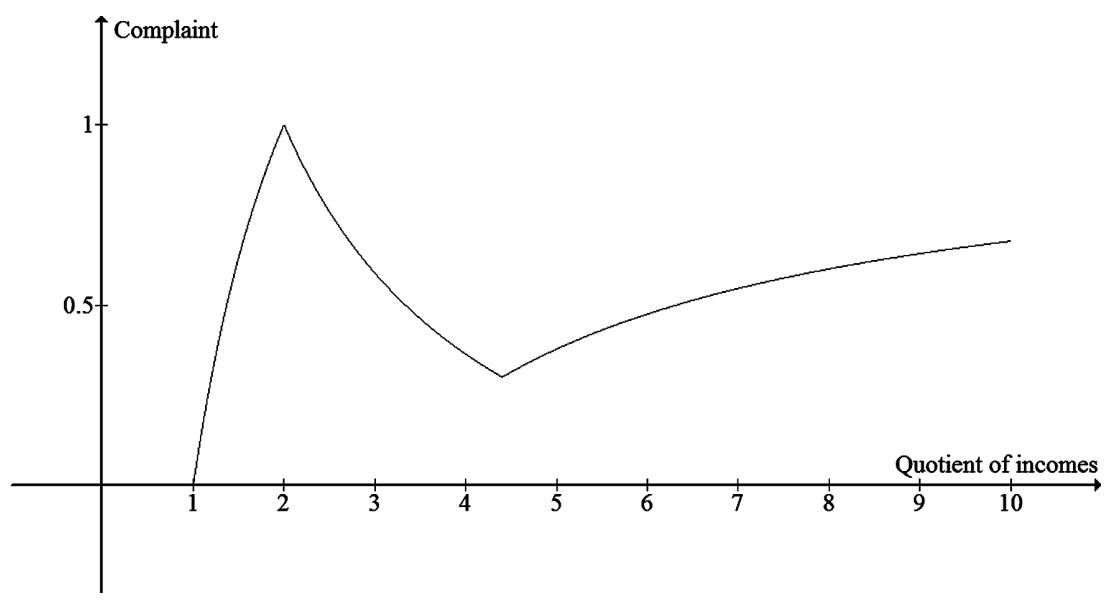

Fig. 1. Relation between extent of complaint and quotient of incomes for $\alpha=0.7$ and $\beta=2$.

Such complaint function (and every other, complying with assumption 5), violates the transfer (Pigou-Dalton) principle. 
Despite the fact that this rule is recognized as fundamental in inequality measurement - see Cowell and Ebert (2004, p. 74), Podder (1996, p. 365) - it seems to have very little support in empirical (questionnaire-based) research on axiomatic foundations of this measurement - see Amiel (1999, p. 234), Devoogh (2003). That is why, in some recent studies - for instance Cowell and Ebert (2004, p. 7477) - there is made a distinction between different formulations of Pigou-Dalton principle. Although, from the point of view of measurement of complaints in the context of reference groups, none of the versions of that principle should be treated as absolutely binding. It is quite obvious that dividing population into some groups may result (especially in case of transfers between these groups) in inconsistency with that rule.

\section{2) Aggregation for an individual}

The second stage comprises aggregation of formerly described complaints so as to assess an overall level of dissatisfaction that is felt by an individual with respect to income inequality (distribution of income within the population). To enable this aggregation the subsequent assumption has to be made.

Assumption $6^{*}$. Overall complaint of a given person is the sum of

a. Single complaints, felt with respect to all persons in the reference group which are better-off (with higher income). Every such complaint is attached the same weight which equals one.

b. Complaint felt with respect to average income in the reference category. Extent of this complaint is assessed for median income (positional measures are better adjusted to perception of diversification among higher incomes than their classical counterparts) and attributed a weight equal to number of people in the reference category.

In both subpopulations it is assumed that all differences in perception of inequality are captured by complaint function. 
Special significance of people from the reference group could be additionally reflected through appropriate weighting scheme. In such a case weights within reference group should be higher than in reference category, but this will not be further analyzed in the paper.

The measurement of complaint in reference category is inspired by results of Devoogh (2003). He analyzed the empirical relevance of Temkin's theory and found out that most of respondents chose the average income as a point of reference for assessment of own income and - consequently - the extent of complaint: $75.2 \%$ in the 'verbal part', where respondents were to choose between concepts of inequality measurement distinguished by Temkin (1986).

However, Devoogh did not analyze the case of more than one subpopulation. Therefore, because of assumptions concerning importance of the reference group, average income as a point of reference could be used only for reference category.

Then, aggregated complaint of the individual $i$ is given by formula:

$$
i C(i, \mathbf{x}, \alpha, \beta)=\sum_{k=i+1}^{m} C\left(x_{i}, x_{k}, \alpha, \beta\right)+(n-m) C\left(x_{i}, M e\left(x_{m+1}, \ldots, x_{n}\right), \alpha, \beta\right)
$$

where $i$ denotes the number of individual, $\mathbf{x}$ is a vector of incomes in population (in nondecreasing order $x_{1} \leq \ldots \leq x_{i} \leq \ldots$ $\leq x_{m} \leq \ldots \leq x_{n}$ ), $n$ denotes the size of population (vector $\mathbf{x}$ ) and $m$ is a number of the best-off person in the reference group of person $i\left(x_{m} \leq \beta x_{i}\right.$ and $\left.x_{m+1}>\beta x_{i}\right)$.

\section{3) Aggregation for a population}

At the final, third stage, complaints of individuals are aggregated over the whole population. The last two assumptions determine aggregation at this level.

Assumption 7. All individuals in the population have the same complaint function $C$. 
Assumption 8. Aggregate feeling of complaint is the weighted sum of complaints felt by individuals.

Measures of, respectively, aggregate $(a C)$ and mean $(m C)$ complaint in the population are given by the following formulas:

$$
\begin{aligned}
& a C(\mathbf{x}, \alpha, \beta)=\sum_{i=1}^{n} w_{i} \cdot i C(i, \mathbf{x}, \alpha, \beta) \\
& m C(\mathbf{x}, \alpha, \beta)=\frac{2}{n(n-1)} a C(\mathbf{x}, \alpha, \beta)
\end{aligned}
$$

where $w_{i}$ - weights, such that $w_{1} \geq w_{2} \geq \ldots \geq w_{n}$ and $\frac{1}{n} \sum_{i=1}^{n} w_{i}=1$. In particular, all weights may be equal, but results reported by Devoogh (2003) suggest that to the greatest complaints should be attached the highest weights (Temkin's concept of weighted mean has been supported in 'verbal part' by $77.3 \%$ of respondents). Weighting scheme of this type can be given by:

$$
w_{i}=\frac{2(n-i+1)}{n+1}
$$

This weighting scheme is analogous to the one used in Gini coefficient - see Lambert (2001, p. 34) - and to the one proposed by Devoogh (2003).

Weights depend on position of individual's income in income distribution of the whole population. The higher the income of a given person is, the lower weight is attributed to his/her complaint. This scheme results from implicit assumption that lower incomes (generating higher complaints) should be the center of attention of all those that are concerned with issue of inequality. In case of presented complaint function, requirement of such reverse relationship is not satisfied in some exceptional instances. The opposite situation may occur, for example, when 2 individuals - say A and B - possess incomes that differ only slightly (B's income is higher) while the rest of population (suitably large) is 
on an income, for which complaint function of person A reaches the local minimum. However, such exceptions seem not to be important from the point of view of general analysis and will not be taken into consideration.

\section{RELATIVE DEPRIVATION, INEQUALITY AND INCOME TAXATION}

The way of relative deprivation measurement, presented in previous sections, leads to conclusions that are only partially in conformity with those, arising from analysis of inequality. Although the optimal situation is in both cases the same - absolutely equal distribution of income (wealth) guarantees lack of complaints as well as inequality - significant differences concern certain conditions, on which both inequality and aggregate complaint attain maximum. Inequality is maximal, when all the property (income, wealth) is owned by one person in the population. In case of relative deprivation there is no such univocal answer. When relative deprivation is measured in the manner analogous to inequality - see, for example, Pedersen, (2004) conclusions are the same. But overruling some assumptions - especially principle of transfers - results in disparate conclusions. That is, for instance, the case of concept proposed by Podder (1996). He suggests that equal division of population into two groups: 'haves' and 'have-nots', results in maximal extent of relative deprivation and may - in his opinion - even provoke rebellion.

Also in the approach presented in this paper, distribution that maximizes aggregate complaint is considerably different from the case of absolute concentration. Just the opposite: significant concentration may cause almost no complaints (that is a consequence of both shape of complaint function, which attains values from zero to one, and adopted weighting schemes). Change in the situation of a single individual - even very significant 
(starting from the prefect equality) - does not, in fact, influence the structure of the reference group of the rest of the population. Continuing this line of thought, it is possible to reach a conclusion that a situation, when there are few groups within the population (consisting of people with exactly equal income), is also nearly optimal with respect to extent of aggregate complaint. However, the worst is the diversification of income in the population. Coefficient of average complaint attains values close to maximum (one), when every successive person in the population (ordered by amount of income) is significantly richer - especially, when income of person $i$ is given by:

$$
x_{1}=\beta^{i-1} x_{1} \text { for } i=2, \ldots, n
$$

where $\beta$ denotes extent of reference group (in income terms) and $x_{1}$ - income (positive) of the worst-off person.

Mentioned differences considerably influence deliberations on tax structures. In terms of complaints (just the opposite than in terms of inequality, when concavity of income evaluation function and additively separable form of social welfare function are assumed), tax progression could not be treated as property that unconditionally improves situation in population. Violation of transfer principle in construction of the measure of relative deprivation allows to indicate another path for improvement in fiscal system. Instead of decreasing in overall inequality in the population (what could be seen as one of the main aims of progressive tax systems), it suggests stratification without restrictions on inequality level. It results in the tax system that is mostly concerned with groups and inequality within them.

Schedule of 'group-based thresholds' could be given as an example of such system. Such schedule would consist of set of thresholds, established in the manner analogous to the one from equation (8). However, instead of $\beta$ (denoting extent of reference group), value, for which complaint function attains the local 
minimum, should be used as a multiplier. And a tax duty would be calculated as a difference between gross income and the closest lower threshold.

Undoubtedly, it is impossible to imagine such a system as actual solution: man could expect very large scale of evasion and appropriate adjustments in the reported taxable income. Mainly, because of the fact that it is oversimplified to construct a whole tax system from the point of view of the single aspect. Nonetheless, this model may indicate a direction for transformations of actual tax systems or define criterion for assessment of expected (or already implemented) changes. However, such appraisals could be reliable only for marginal changes.

The fundamental feature of presented approach, in the context of tax system optimization, is that it allows existence of very large asymmetry in the after-tax income (empirical evidence confirms that distribution of net income - although less unequal than of gross income - is very far from equality). Moreover, what is quite surprising for analysis of relative deprivation, there are no theoretical basis for statement that marginal tax rates should be higher for higher incomes. In above characterized, theoretical tax system, minimal and maximal possible effective tax rates are equal for all tax brackets (average tax rates depend on reported income within the reference group).

Additionally, what should be emphasized, such tax system, even in its radical, theoretical version, is consistent with notion of private property (even though, as every other tax system, restricts it in a sense), which - in fact - constitutes essence of inequality. Since complete elimination of inequality would result in elimination of private property and freedom - see Zaleski (2003, p. 110-113). 


\section{CONCLUSIONS}

To conclude, it should be stated that relative deprivation constitutes only a part of much broader issue - social justice. And social justice seems to be the appropriate framework that should be considered, when analyzing problem of unequal distribution of wealth. Certainly, this paper is in no way aimed to redefine notion of social justice or arbitrate the dispute about optimal redistribution and consequences of inequality. Nevertheless, from the point of view of relative deprivation, seen as aggregation of complaints in the context of reference groups, overall inequality itself seems not to be of a crucial importance for perception of social justice. It does not mean that issue of inequality should not be taken into account while analyzing situation of society the only suggestion is that optimal inequality level is a secondary problem in relation to some more general assumptions.

That is why, a tax system, resulting from the presented concept of complaints, does not aim at limiting inequality - choice of the appropriate level of this parameter should take into account - first of all - model of society. And tendency to restricting inequality seems to be closely associated - among others - with democracy. However, as can be learned from history, some social systems had stably existed despite very high inequality level.

\section{REFERENCES}

Amiel, Y. (1999). The Measurement of Income Inequality: The Subjective Approach, in: Jacques Silber (ed.), Handbook of Income Inequality Measurement. Dordrecht: Kluwer Academic Publishers: 227-241.

Atkinson, A. B. (1970). On the Measurement of Inequality, Journal of Economic Theory. 2: 24463.

Belk, R. W. (1985). Materialism: Trait Aspects of Living in the Material World, Journal of Consumer Research. 12: 265-280. 
Cowell, F. A. (2000). Measurement of Inequality, in: Anthony B. Atkinson and Francois Bourguignon (eds.), Handbook of Income Distribution, vol. 1. Amsterdam: Elsevier Science: 87-166.

Cowell, F. A., Ebert, U. (2004). Complaints and inequality, Social Choice and Welfare. 23: 71-89.

Devoogh, K. (2003). Measuring Inequality by Counting 'Complaints': Theory and Empirics, Economics and Philosophy. 19: 241-263.

Dawson, M. E., Chatman E. A. (2001). Reference Group Theory with Implications for Information Studies: a Theoretical Essay, Information Research. 6(3) available at: http:/ /informationr.net/ir/6-3/paper105.html

Grusky, D., Kanbur, G. (2005). Conceptual Ferment in Poverty and Inequality Measurement: The View from Economics and Sociology, in: David Grusky and Ravi Kanbur (eds.), Poverty And Inequality: Essays By Amartya Sen, Martha C. Nussbaum, François Bourguignon, William J. Wilson, Douglas S. Massey, And Martha A. Fineman. Stanford: Stanford University Press (forthcoming). available at: http://www.arts.cornell.edu/poverty/kanbur/GruskyKanbur.pdf

Lambert, P. J. (2001). The distribution and redistribution of income. Manchester: Manchester University Press.

Merton, R. K. (1957). Social Theory and Social Structure. New York: The Free Press. Polish edition: Warszawa: PWN 2002.

Pedersen, A.W. (2004). Inequality as Relative Deprivation. A Sociological Approach to Inequality Measurement, Acta Sociologica. 47(1): 31-49.

Podder, N. (1996). Relative Deprivation, Envy and Economic Inequality, Kyklos. 49: 353-376.

Rawls, J. (1971). A Theory of Justice. Cambridge: Harvard University Press.

Runciman, W. G. (1966). Relative Deprivation and Social Justice: A Study of Attitudes to Social Inequality in Twentieth-Century England. London: Routledge \& Kegan Paul.

Sen, A. K. (1973). On Economic Inequality. Oxford: Oxford University Press.

Temkin, L. S. (1986). Inequality, Philosophy and Public Affairs. 15: 99-121.

Temkin, L. S. (1993). Inequality. Oxford: Oxford University Press.

Yitzhaki, S. (1998). More than a Dozen Alternative Ways of Spelling Gini, Research on Economic Inequality. 8: 13-30.

Zaleski, Z. (2003). Psychologia własności i prywatności (Psychology of Property and Privacy). Warszawa: Wydawnictwo Akademickie Żak (in Polish). 